\title{
Synthesis of Polyaniline/Graphene Oxide/Azobenzene Composite and Its Adjustable Photoelectric Properties
}

\author{
Yanhua Teng $\left(\mathbb{D}\right.$, Shiqin Li, Changguo Xue $\mathbb{D}$, Hongyan Zhang $\mathbb{D}^{D}$, Lingkai Zhu, and Yu Tang \\ School of Material Science and Engineering, Anhui University of Science and Technology, Anhui, Huainan 232001, China \\ Correspondence should be addressed to Changguo Xue; chgxue@foxmail.com
}

Received 9 October 2019; Revised 22 January 2020; Accepted 20 February 2020; Published 16 March 2020

Guest Editor: Zhiyuan Qi

Copyright ( $\odot 2020$ Yanhua Teng et al. This is an open access article distributed under the Creative Commons Attribution License, which permits unrestricted use, distribution, and reproduction in any medium, provided the original work is properly cited.

\begin{abstract}
Azobenzene derivatives have fast light response characteristics; in this paper, a new azobenzene derivative (Azo) was synthesized and to be made a composite (PANI/GO/Azo) with polyaniline/graphene oxide (PANI/GO). Both composites were carefully investigated by scanning electron microscopy (SEM), Fourier transform infrared spectroscopy (FT-IR), and thermogravimetric analysis (TGA). Moreover, their electrochemical properties were characterized by the electrochemical workstation, including cyclic voltammetry (CV), galvanostatic charge-discharge (GCD), cycling stability, and electrochemical impedance spectroscopy (EIS). The results demonstrated that the PANI/GO/Azo composite has a higher capacitance of $478.3 \mathrm{~F} \mathrm{~g}^{-1}$ than that of PANI/GO $\left(359.9 \mathrm{~F} \mathrm{~g}^{-1}\right)$ at a current density of $1 \mathrm{~A} \mathrm{~g}^{-1}$. PANI/GO/Azo composite showed excellent photosensitive electrochemical properties under UV irradiation, and its rate of capacitance change achieved about 52.57\%. Additionally, the PANI/GO/Azo composite also displayed high reversibility, with specific capacitance retention of $92 \%$ after 500 cycles. Therefore, the PANI/GO/Azo electrode with a controllable electrochemical performance by UV irradiation had a great potential in the photoresponsive supercapacitor.
\end{abstract}

\section{Introduction}

Recently, with the growing trend about solar energy, wind energy, and other clean energy generations, the development of alternative storage resources is very indispensable. Supercapacitors have attracted considerable interest in electronic energy storage systems due to low cost, simple charging circuit, long cycle life, and high power density [1-4]. The performance of supercapacitors is closely related to electrode materials. Therefore, a large number of theoretical and experimental research studies have been focused on designing and preparing novel and promising electrode materials with high capacitances and long-term stability [3-8].

Electrode materials of supercapacitors are mainly divided into three categories: conducting polymers, carbon materials, and transition metal oxides [3]. Among a large number of conducting polymers, polyaniline (PANI) is the most widely used electrode material owing to its advantages of simple synthesis process, excellent stability, and high electrical conductivity [9-11]. However, the poor cycling stability and lower surface area of PANI limit its applications in supercapacitors $[12,13]$. Significant attention has been focused on integrating PANI with high-conductive carbon materials to obtain high-performance composite electrode materials. Among a large number of carbon materials, graphene oxide (GO) [14] is widely studied to make composites with PANI because of its relatively large surface area [15], good flexibility, and excellent mechanical properties $[5,6,16,17]$. For example, Manoj et al. prepared PANI/GO nanocomposites by in situ oxidative polymerization technique, which showed specific capacitance $\left(132 \mathrm{Fg}^{-1}\right.$ at $5 \mathrm{Ag}^{-1}$ ) with excellent capacity retention over 1,000 cycles [1]. Zhao et al. synthesized vertically oriented PANI/GO nanocomposites by interfacial polymerization, which had a specific capacitance $\left(369 \mathrm{~F} \mathrm{~g}^{-1}\right.$ at $\left.1 \mathrm{~A} \mathrm{~g}^{-1}\right)$ and exhibited capacitance retention of $81.2 \%$ after 500 cycles at $10 \mathrm{Ag}^{-1}$ [18]. Li et al. reported that the specific capacitance decreased $9 \%$ of PANI/ GO electrode materials after 1,000 cycles compared with initial capacitance [19]. Alternatively, some researchers prepared various structures of PANI/GO composites such as three-dimensional porous structure [20], sandwiched structure [21], hollow spheres [22], and sheet-like morphology [9] 
to improve their electrochemical properties. The electrochemical properties of PANI/GO electrode materials were remarkably improved in those ways. However, their electrical properties could not be real-time adjusted.

Therefore, the design of electrode materials with excellent and real-time controllable electrical properties has considerable prospects. As a kind of clean energy, light can be easily and quickly controlled remotely [23]. Using light to regulate the properties of composites has become a research hotspot in the field of science [24-28]. Azobenzene (Azo) compound is the well-known light-induced material owing to its unique characteristics, including widely applicable performance, photochemical stability, and fast response [29, 30]. It can undergo trans-cis isomerization by performing ultraviolet/visible light irradiation [31] or heating $[32,33]$. Due to the unique property, Azo is often used as a photoswitchable molecule [26, 33-37]. Meanwhile, there have been some researchers who studied surfactant-modified graphene hybrids containing azobenzene groups and regulated their electrochemical performance $[1,26,38-40]$. However, to the best of our knowledge, there is no report in the literature about obtaining adjustable composite electrode materials by modification of the PANI/GO composite with azobenzene. As a result, combining Azo with the PANI/ GO composite to prepare adjustable ternary hybrid electrode materials is promising.

In this article, a new photosensitive azobenzene derivative was prepared by the diazotization method as additive. With GO/PANI as the matrix, the one-step method is used to fabricate the adjustable composite electrode materials-PANI/GO/Azo composite. PANI/GO/Azo composite has a higher capacitance of $478.3 \mathrm{~F} \mathrm{~g}^{-1}$ than that of PANI/ GO $\left(359.9 \mathrm{Fg}^{-1}\right)$ at a current density of $1 \mathrm{Ag}^{-1}$. Both composites were carefully studied and analyzed.

\section{Materials and Methods}

2.1. Chemicals. The chemicals were used without further purification. Aniline $\left(\mathrm{C}_{6} \mathrm{H}_{8} \mathrm{~N}, 99.5 \%\right)$, graphene oxide aqueous solution $(\mathrm{GO})$, ammonium persulfate $\left(\left(\mathrm{NH}_{4}\right)_{2} \mathrm{~S}_{2} \mathrm{O}_{8}\right.$, $98 \%)$, acetone $\left(\mathrm{CH}_{3} \mathrm{COCH}_{3}, 99.5 \%\right)$, hydrochloric acid ( $\mathrm{HCl}, 35 \%)$, p-toluidine $\left(\mathrm{C}_{7} \mathrm{H}_{9} \mathrm{~N}, 99 \%\right)$, sodium hydroxide $(\mathrm{NaOH}, 96 \%)$, ethyl alcohol absolute $\left(\mathrm{C}_{2} \mathrm{H}_{6} \mathrm{O}, 99.7 \%\right)$, potassium iodide (KI, 99\%), 6-chloro-1-hexanol $\left(\mathrm{C}_{6} \mathrm{H}_{13} \mathrm{ClO}\right.$, $98.5 \%)$, potassium carbonate $\left(\mathrm{K}_{2} \mathrm{CO}_{3}, 99 \%\right)$, thiourea $\left(\mathrm{CH}_{4} \mathrm{~N}_{2} \mathrm{~S}, 99 \%\right)$, etrahydrofuran (THF, 99\%), phenol $\left(\mathrm{C}_{6} \mathrm{H}_{5} \mathrm{OH}, 99 \%\right)$, sodium nitrite $\left(\mathrm{NaNO}_{2}, 99 \%\right)$, and $1,2-$ dibromoethane $\left(\mathrm{BrCH}_{2} \mathrm{CH}_{2} \mathrm{Br}, 99 \%\right)$ were purchased from Sinopharm Chemical Reagent Co. All water used in this experiment is deionized water.

2.2. Synthesis of Azobenzene. 2-((6-(4-(p-Tolyldiazenyl)phenoxy)hexyl)oxy)ethane-1-thiol $\left(\mathrm{CH}_{3}-\mathrm{C}_{6} \mathrm{H}_{4}-\mathrm{N}=\mathrm{N}-\mathrm{C}_{6} \mathrm{H}_{4}-\mathrm{O}-\mathrm{C}_{6} \mathrm{H}_{12}-\mathrm{O}-\mathrm{C}_{2} \mathrm{H}_{4}-\mathrm{SH}\right)$ was synthesized according to the literature of Weis et al. [41] and Haiying and Zhongfan [42]. ${ }^{1} \mathrm{H}$ NMR (400 MHz, chloroform-d) $\delta$ 7.93-7.84 (m, 2H, Ar-H), 7.82-7.74 (m, 2H, Ar-H), 7.33-7.25 (m, 2H, Ar-H), 7.03-6.94 (m, 2H, Ar-H), $4.04(\mathrm{t}, J=6.5 \mathrm{~Hz}$, $2 \mathrm{H}, \mathrm{Ar}-\mathrm{O}-\mathrm{CH}_{2}$ ), 3.67 (t, J=6.5 Hz, $2 \mathrm{H}, \mathrm{SH}-\mathrm{CH}_{2} \mathrm{CH}_{2}-\mathrm{O}$ ), 4.39- $4.35\left(\mathrm{t}, J=6.5 \mathrm{~Hz}, 2 \mathrm{H}, \mathrm{SH}-\mathrm{CH}_{2}\right) 2.42\left(\mathrm{~s}, 3 \mathrm{H},-\mathrm{CH}_{3}\right), 1.84(\mathrm{dq}$, $\left.J=7.9,6.5 \mathrm{~Hz}, 2 \mathrm{H},-\mathrm{O}-\mathrm{CH}_{2}-\mathrm{CH}_{2}\right), 1.68-1.39\left(\mathrm{~m}, 8 \mathrm{H},-\mathrm{CH}_{2^{-}}\right.$ $\mathrm{CH}_{2}-\mathrm{CH}_{2}-\mathrm{CH}_{2}$ ) (Figure 1).

2.3. Synthesis of the PANI/GO/Azo Composite. $1.2 \mathrm{mmol}$ of Azo was dissolved in $50 \mathrm{~mL}$ ethanol and kept ultrasonic for 15 minutes. $3.8 \mathrm{~mL}$ GO solution, which had been dispersed, was added to $140 \mathrm{~mL}$ deionized water and kept ultrasonic for $15 \mathrm{~min}$. The above two solutions were poured into a threenecked flask. Then, the mix solution was stirred quickly at $0^{\circ} \mathrm{C}$. After $10 \mathrm{~min}$, aniline $(1.85 \mathrm{~mL})$ and concentrated hydrochloric acid $(5 \mathrm{~mL})$ were added slowly to the threenecked flask in turn, and the mixture solution was freed from oxygen by bubbling with high purified nitrogen for $30 \mathrm{~min}$. Next, the ammonium persulfate solution was added slowly to the mixture solution. The whole reaction process was kept another $7 \mathrm{~h}$ accompanying vigorous stirring at $0^{\circ} \mathrm{C}$. Finally, the precipitate was filtered, and the filtered cake was washed with deionized water and ethyl alcohol absolute several times and dried under vacuum overnight at $60^{\circ} \mathrm{C}$. Additionally, the PANI/GO composite without Azo was prepared under the same condition as the control group.

2.4. Material Characterization. Proton nuclear magnetic resonance $\left({ }^{1} \mathrm{HNMR}\right)$ spectrum of Azo was recorded on a BRUKER ARX400 MHz spectrometer using chloroform- $d$ as a solvent with tetramethylsilane (TMS) as the internal standard at room temperature. FT-IR was recorded using a NICOLET380 FT-IR spectrometer (Thermo Scientific, US). The photochemical behavior of Azo was characterized by UV-Vis absorption spectroscopy (UV-2600, Yuanxi Ltd, Shanghai). The apparent morphology of the hybrids was examined by SEM (S-3400N, Hitachi Ltd, JP). TGA was carried out using a thermogravimetric analyzer (S-3000N, Hitachi Ltd, JP) at the heating rate of $10^{\circ} \mathrm{C} / \mathrm{min}$ in the $\mathrm{N}_{2}$ atmosphere.

2.5. Electrochemical Measurements. Cyclic voltammetry $(\mathrm{CV})$, galvanostatic charge-discharge (GCD), and electrochemical impedance spectroscopy (EIS) were performed on a CHI660E (Shanghai Chen Hua, China) workstation using a standard three-electrode cell setup. The electrode which is coated with the sample, platinum wire, and saturated silver chloride electrode $(\mathrm{Ag} / \mathrm{AgCl})$ was used as a working electrode, opposite electrode, and reference electrode, respectively. $1 \mathrm{M} \mathrm{H}_{2} \mathrm{SO}_{4}$ aqueous solution was used as the electrolyte. The preparation process of the working electrode is shown in Figure 2. A stainless steel mesh sheet of $7 \mathrm{~cm} \times 1.5 \mathrm{~cm}$ was soaked in ethanol for $2 \mathrm{~h}$ and then soaked in deionized water with ultrasonic for $0.5 \mathrm{~h}$. After that, it was put into an oven for drying at $60^{\circ} \mathrm{C}$ for $5 \mathrm{~h}$. After that, it marked the mass of stainless steel mesh as $m_{0}$. N-Methyl pyrrolidone and polyvinylidene fluoride were proportionally configured into dispersion solution with ultrasonic for $10 \mathrm{~min}$, and then the sample was proportionally configured into uniform solution required for testing. The prepared solution was spread on the electrode plates evenly, and then 


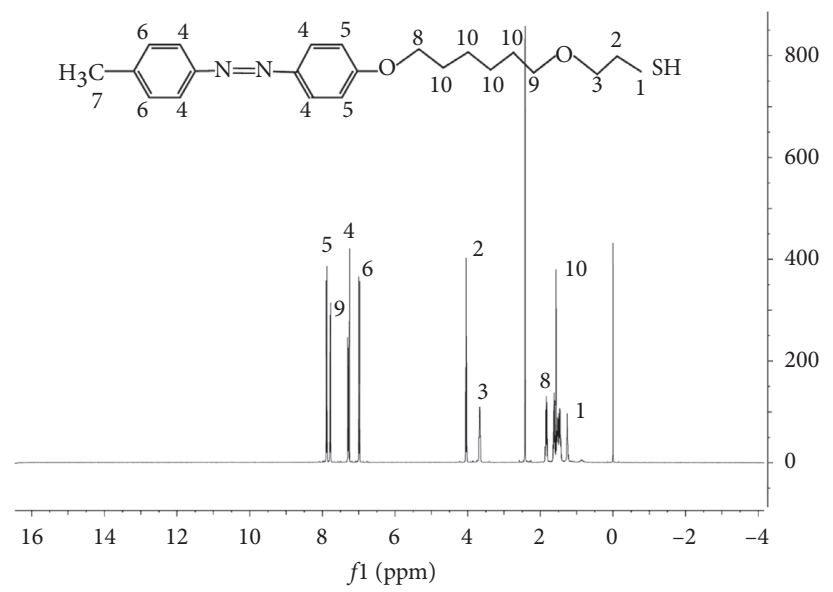

Figure 1: ${ }^{1} \mathrm{H}$ NMR spectra of Azo in chloroform-d $\left(\mathrm{CDCl}_{3}\right)$.

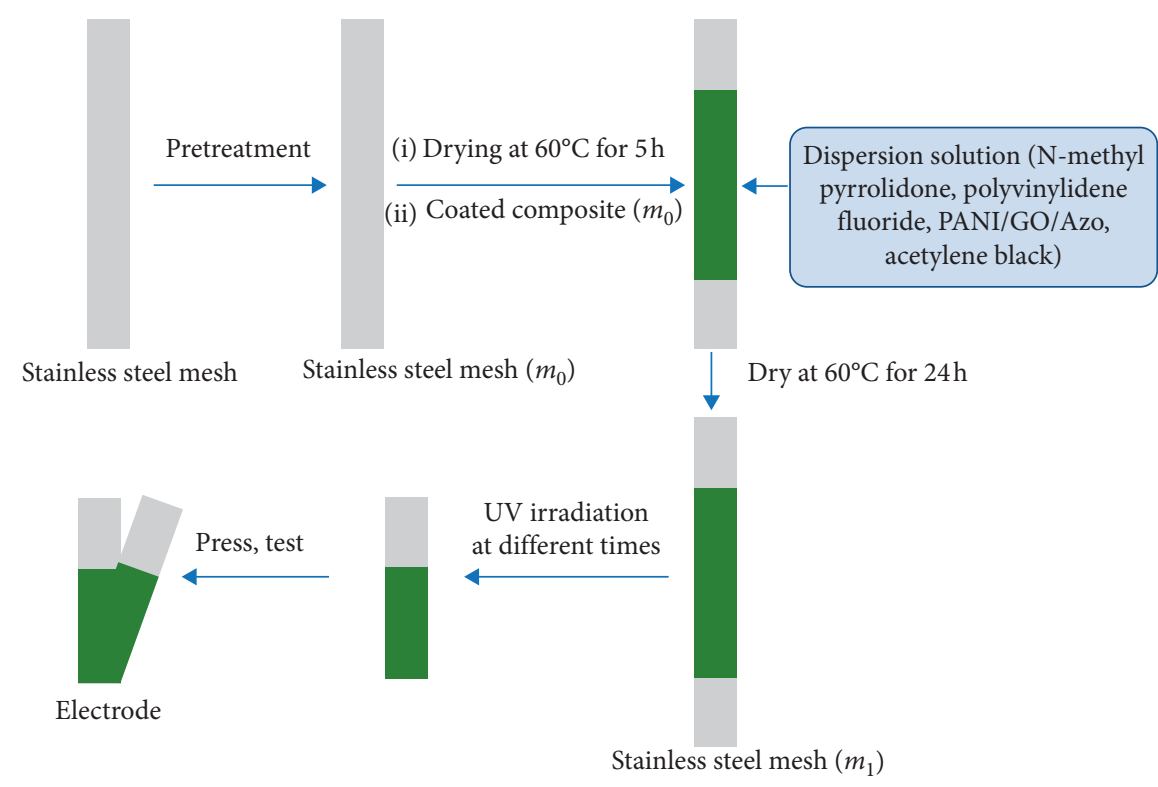

FIGURE 2: Illustration of the process for the preparation of the PANI/GO/Azo electrode.

dried them at $60^{\circ} \mathrm{C}$ for $24 \mathrm{~h}$. Finally, it was weighed again (marked as $m_{1}$ ), pressed, and tested.

\section{Results and Discussion}

\subsection{Identification and Characterization}

3.1.1. UV-Vis Absorption Spectra Analysis. As shown in Figure 3, the UV-Vis spectra of Azo at different times upon irradiation with light at $365 \mathrm{~nm}$ have been investigated. When irradiated by the UV lamp, the azobenzene group was isomerized from trans to cis conformer (Figure 3(b)). The absorbance band corresponding to the $\pi-\pi^{*}$ transition at $348 \mathrm{~nm}$ decreases remarkably, and at the same time, for the absorbance band corresponding to the $n-\pi^{*}$ transition at $450 \mathrm{~nm}$ increases lightly in Figure 3(a) [27, 37]. After UV irradiation for $30 \mathrm{~min}$, the UV-Vis spectra of Azo do not change anymore, indicating azobenzene groups have changed to a cis saturated state. This photoisomerization is reversible; after UV irradiation, Azo slowly recovers to its initial absorption feature when it is kept in the dark for five days. The result indicates that Azo has remarkable photosensitive properties.

3.1.2. FT-IR Analysis. The FT-IR spectra of Azo, PANI/GO, and PANI/GO/Azo are shown in Figure 4. The spectrum of Azo showing peaks at $1592 \mathrm{~cm}^{-1}$ was assigned to $\mathrm{N}=\mathrm{N}$ stretching. In the case of the FT-IR spectrum of PANI/GO, the vibrations at $3436,1744,1571,1481$, and $1119 \mathrm{~cm}^{-1}$ were consistent with $\mathrm{N}-\mathrm{H}$ stretching vibration, $\mathrm{C}=\mathrm{O}$, quinoid ring stretching vibration, benzene ring stretching vibration, and $\mathrm{C}-\mathrm{N}$ peak [43]. These results indicate that the PANI/GO composite was synthesized successfully. Besides, the spectrum of PANI/GO/Azo is essentially the same as that of PANI/GO except for the peak centered at $1600 \mathrm{~cm}^{-1}$, 


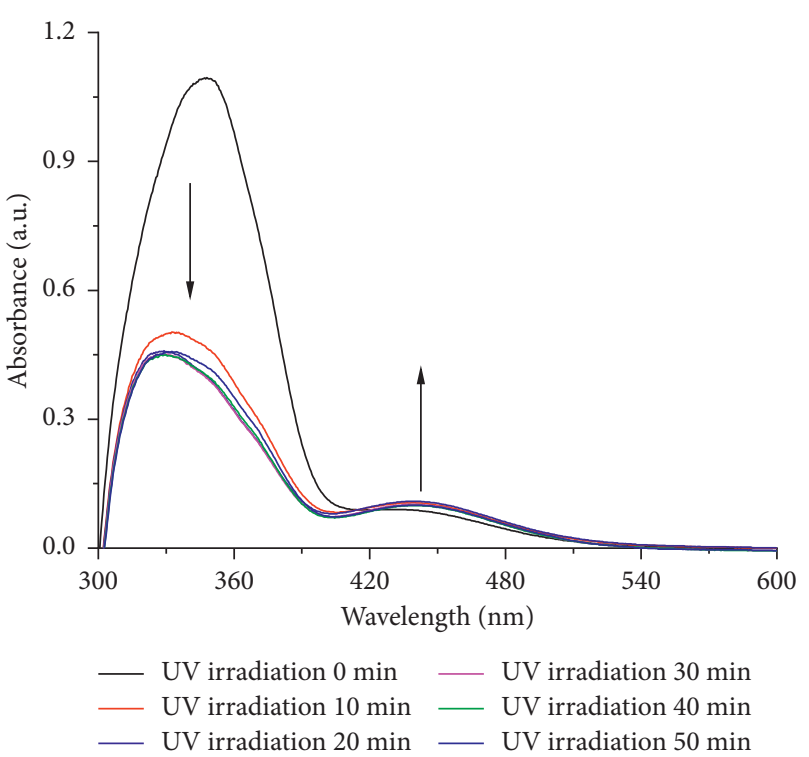

(a)

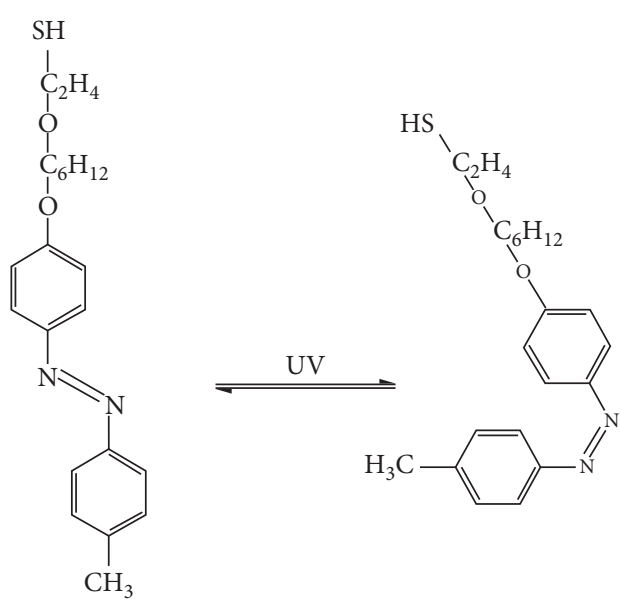

(b)

Figure 3: (a) UV-Vis spectra of Azo in chloroform $\left(\mathrm{CDCl}_{3}\right)$ solution; (b) the structure of Azo and photoinduced isomerization upon UV light $(365 \mathrm{~nm})$ irradiation.

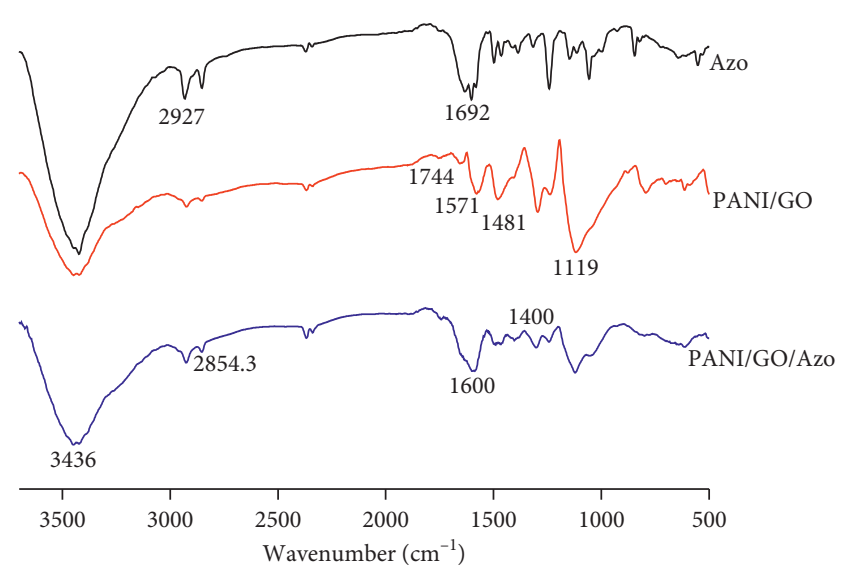

Figure 4: FT-IR spectra of Azo, PANI/GO, and PANI/GO/Azo.

which can be attributed to the superposition of $\mathrm{N}=\mathrm{N}$ stretching vibrations and benzene ring stretching vibration, while compared with Azo IR spectra, the peaks of the PANI/GO/Azo composite were smaller and redshifted, which indicated that Azo had been successfully attached to PANI/GO.

3.1.3. SEM Analysis. SEM observes the morphologies of $\mathrm{PANI} / \mathrm{GO}$ and PANI/GO/Azo hybrid. PANI/GO presents a deep layer with a pore structure, and there are many fold structures and agglomerations on the surface of the layer (Figure 5(a)). Compared with the SEM structure diagram of PANI/GO/Azo, the latter structure lamella is relatively thin and structured, with smaller particles attached to the surface of the layer. Besides, the whole structure is more compact, and the degree of agglomeration is improved. As shown in
Figure 5(b), the addition of Azo can change the structure of PANI/GO to a certain extent.

3.1.4. TGA Analysis. The thermogravimetric analyses of PANI/GO and PANI/GO/Azo are shown in Figure 6. Three significant stages of weight loss have been observed for the PANI/GO composite. The first stage, located in the range $20 \sim 100^{\circ} \mathrm{C}$, was attributed to the loss of moisture; the second stage was related to decomposition of branched chains of the polymer framework in the temperature range $200 \sim 400^{\circ} \mathrm{C}$; and the last stage, in the temperature $500 \sim 600^{\circ} \mathrm{C}$, the polymer backbone starts to break. Compared to PANI/GO, it can be seen that there are four stages of weight loss which have been observed for the PANI/GO/Azo composite. Similar to the former, at $20 \sim 120^{\circ} \mathrm{C}$, thermal weight loss is about $10 \%$ due to the loss of moisture. The decomposition rate of branched chains is significantly accelerated at $200 \sim 300^{\circ} \mathrm{C}$, and the decomposition rate reaches the maximum value at about $250^{\circ} \mathrm{C}$. Around $360^{\circ} \mathrm{C}$, it was attributed to the decomposition of the Azo backbone. In the temperature $480 \sim 600^{\circ} \mathrm{C}$, polymer backbone chain breakage occurs. The final residue of PANI/GO and PANI/GO/Azo was $50 \%$ and $30 \%$ at $800^{\circ} \mathrm{C}$, respectively. PANI/GO/Azo has a more intensely decomposition degree and worse thermal stability than GO/PANI. These results indicate that Azo has been successfully attached to PANI/GO, and the addition of Azo can change the structure of materials.

\subsection{Electrochemical Properties}

3.2.1. CV Analysis. The electrochemical performances of $\mathrm{PANI} / \mathrm{GO}$ and PANI/GO/Azo composites were measured in a standard three-electrode cell setup in the potential range of $0.12-0.8 \mathrm{~V}$ in $1.0 \mathrm{M} \mathrm{H}_{2} \mathrm{SO}_{4}$ solution. Figure 7 (a) presents $\mathrm{CV}$ 


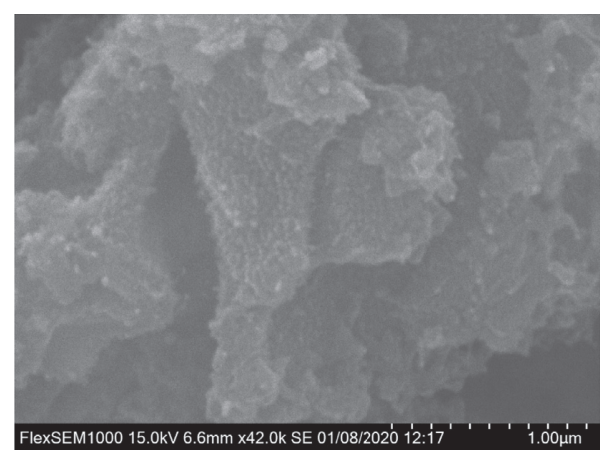

(a)

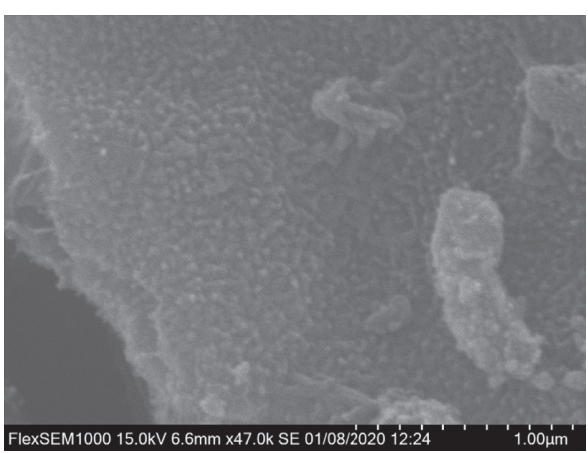

(b)

Figure 5: SEM images of (a) PANI/GO and (b) PANI/GO/Azo.

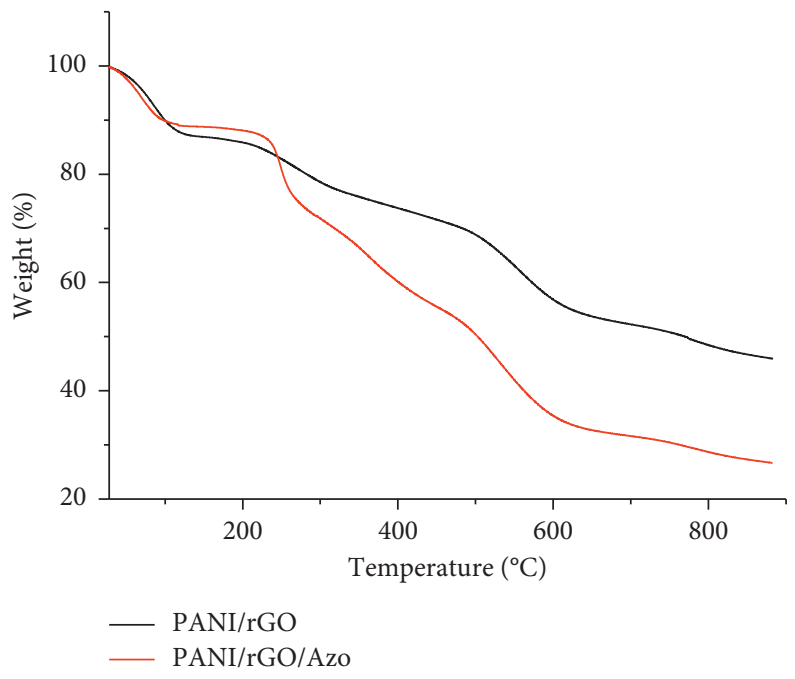

Figure 6: TGA curves of PANI/GO and PANI/GO/Azo compositions.

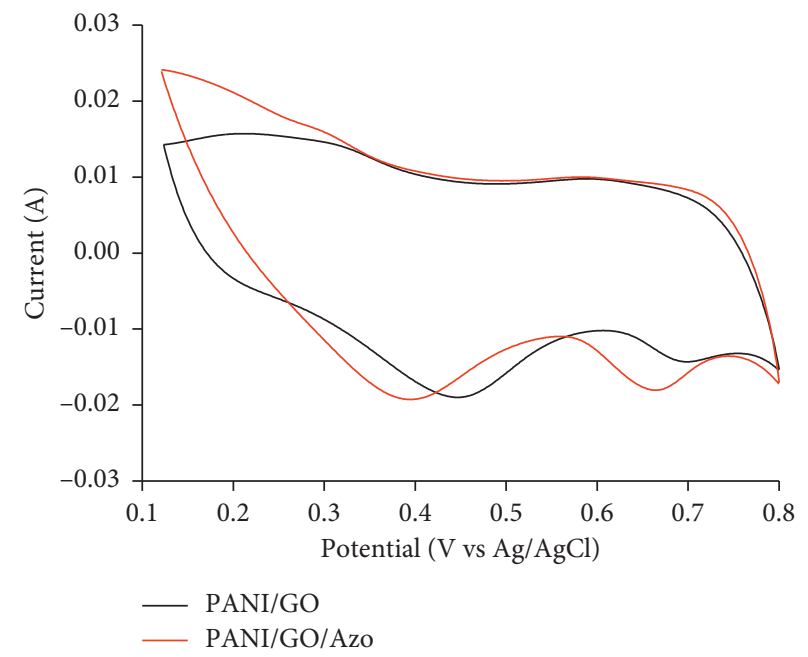

(a)

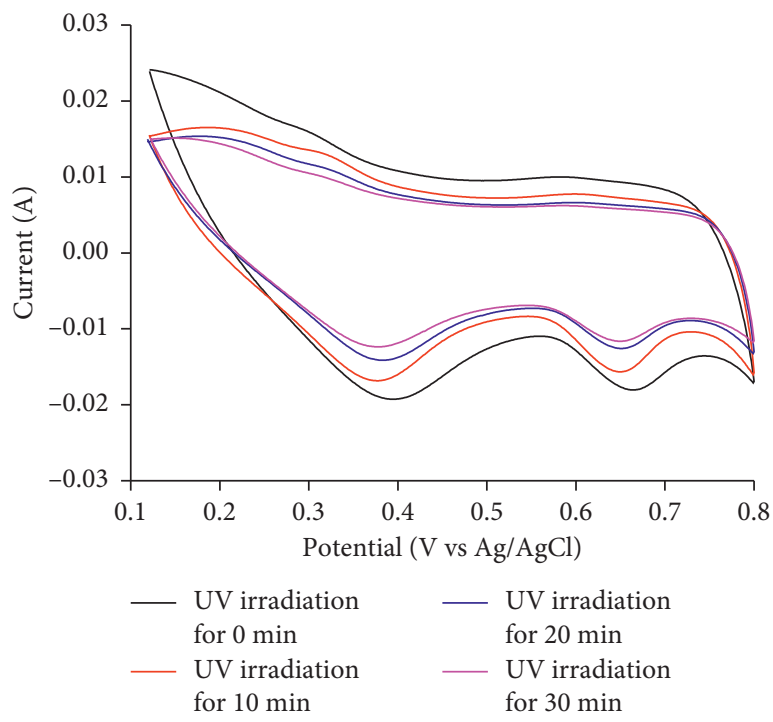

(b)

Figure 7: CV curves of (a) PANI/GO and PANI/GO/Azo composites and (b) PANI/GO/Azo composites at different UV irradiation times at a scan rate of $0.02 \mathrm{~V} / \mathrm{s}$. 


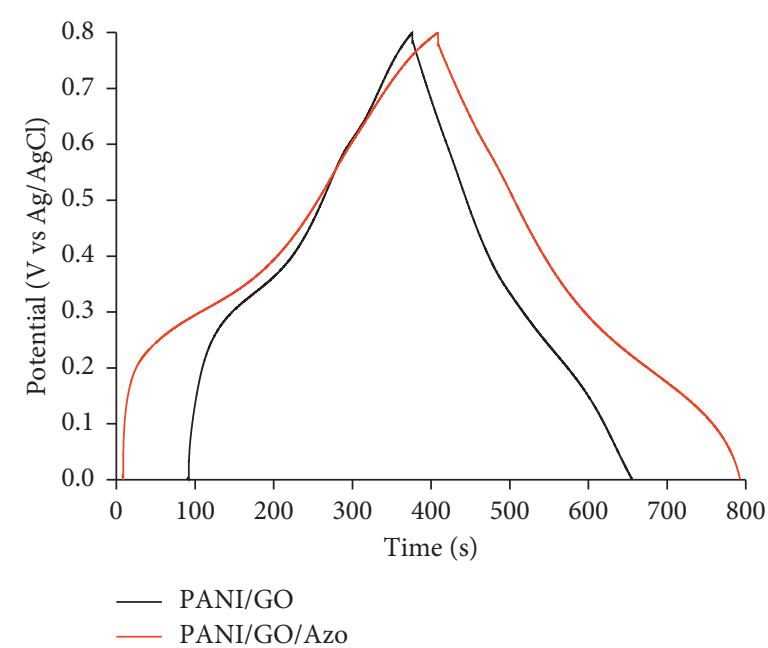

(a)

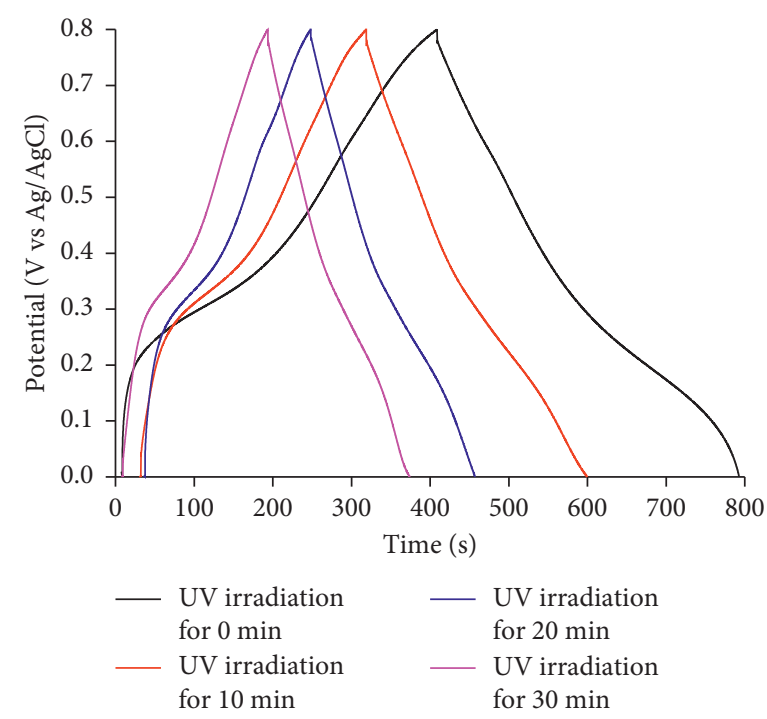

(b)

FIGURe 8: GCD curves of (a) PANI/GO and PANI/GO/Azo composites and (b) PANI/GO/Azo composites at different UV irradiation times.

curves of PANI/GO and PANI/GO/Azo composites, which are measured at a scan rate of $0.02 \mathrm{~V} / \mathrm{s}$. The $\mathrm{CV}$ curves of the PANI/GO electrode show two oxidation peaks and one reduction peak. At the same time, the CV curves of the PANI/GO/Azo electrode show two pairs of redox peaks. Both electrodes exhibit redox peaks indicating the pseudocapacitor behavior of PANI. Besides, we further examined the electrochemical behavior of the photoresponsive PANI/ $\mathrm{GO} /$ Azo hybrid using the $\mathrm{CV}$ test, which is displayed in Figure 7(b). With the increase of UV irradiation time, the oxidation peak gradually becomes weaker, and the area of the CV curve decreases gradually. These phenomena suggest that the specific capacitance can be adjusted by the photoresponsive behavior of PANI/GO/Azo due to the reversible conformational change of the Azo between trans and cis forms upon UV irradiation.

3.2.2. GCD Analysis. Figure 8(a) presents GCD curves of PANI/GO and PANI/GO/Azo composites, and Figure 8(b) presents GCD curves of the PANI/GO/Azo composite at different $\mathrm{UV}$ irradiation times, which within range of $0 \mathrm{~V}$ to $0.8 \mathrm{~V}$ at a current density of $1 \mathrm{Ag}^{-1}$. It can be seen that the GCD curves of electrodes have good symmetry. Besides, according to the discharge time, we can find that the specific capacitance of the PANI/GO/Azo electrode reduces gradually with the increase of UV irradiation time, which is in good agreement with the $\mathrm{CV}$ results.

Besides, the specific capacitances $\left(\mathrm{Cs}, \mathrm{Fg}^{-1}\right.$ ) of the samples were calculated according to the following equation:

$$
\mathrm{Cs}=\frac{I \times \Delta t}{m \times \Delta V},
$$

where $I(\mathrm{~A}), \Delta t(\mathrm{~s}), m(\mathrm{~g})$, and $\Delta V(\mathrm{~V})$ are discharge current, time, the mass of active material $\left(m_{1}-m_{0}\right)$ [43], and the potential window in the charge-discharge curve; the
TABLE 1: The calculation results of Cs values of different composites.

\begin{tabular}{lc}
\hline Sample types & Cs $\left(\mathrm{F} \mathrm{g}^{-1}\right)$ \\
\hline PANI/GO & 359.9 \\
PANI/GO/Azo & 478.3 \\
\hline
\end{tabular}

TABLE 2: The calculation results of Cs values of PANI/GO/Azo at different UV irradiation times.

\begin{tabular}{lc}
\hline UV irradiation time $(\mathrm{min})$ & $\mathrm{Cs}\left(\mathrm{F} \mathrm{g}^{-1}\right)$ \\
\hline 0 & 478.3 \\
10 & 354.6 \\
20 & 261.7 \\
30 & 226.86 \\
\hline
\end{tabular}

calculation results of the Cs values of samples are shown in Tables 1 and 2.

According to Tables 1 and 2, it is evident that the addition of Azo can improve the specific capacitances of the PANI/GO composite, and the sample of PANI/GO/Azo without irradiation possessed the largest Cs. Moreover, the specific capacitances of PANI/GO/Azo can be changed by UV irradiation, and the rate of capacitance change achieved about 52.57\%. These results of Tables 1 and 2 may be explained as follows: along with the increase of light time, trans-PANI/GO/Azo changed into cis-PANI/GO/Azo, which bears a relatively small surface area [44]. This reduction of the accessible surface area of PANI/GO/Azo upon UV irradiation makes the electrocatalytic activity lower. Thus, as the UV irradiation time increasing, the capacitance of the PANI/GO/Azo composite decreased gradually. These results indicated that the capacitance of the PANI/GO/Azo composite could be controlled by UV light.

The cycling stability is an essential factor for a wellestablished electroactive material. The cycling stability of the 


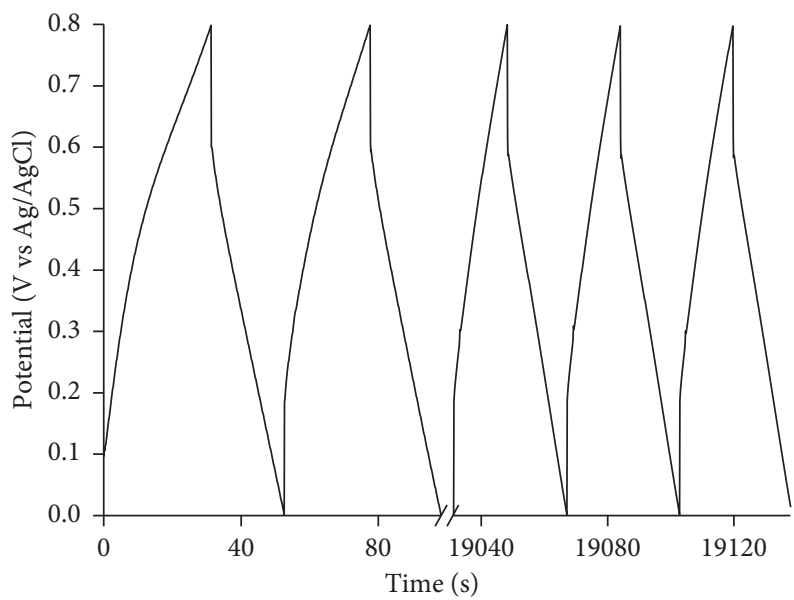

FIgURE 9: The first two and last three cycles of the GCD plots of the PANI/GO/Azo composite measured in the 500 times repeated chargedischarge processes, measured at a current density of $4 \mathrm{Ag}^{-1}$.

PANI/GO/Azo (UV irradiation $0 \mathrm{~min}$ ) electrode was tested by repeatedly measuring the GCD plots at the current density of $4 \mathrm{Ag}^{-1}$ for 500 cycles. Figure 9 shows the GCD curves measured at the first two and last three cycles in the 500 times charge/discharge process. The GCD curves at the first three cycles show similar symmetry to the last three cycles, while the discharge time was reduced. According to equation (1), the specific capacitance of the first cycle about PANI/GO/Azo reaches a value of $103 \mathrm{~F} \mathrm{~g}^{-1}$ under a current density of $4 \mathrm{~A} \mathrm{~g}^{-1}$. After 500 cycles, the specific capacitance reaches a value of around $94 \mathrm{~F} \mathrm{~g}^{-1}$. This corresponds to about $92 \%$ of the initial capacitance, and these results are shown in Figure 10. The capacitance retention remained higher than $90 \%$ during the 500 cycles charge-discharge process. These results suggest that the PANI/GO/Azo composite has high reversibility and excellent cycling stability.

3.2.3. EIS Analysis. EIS of PANI/GO and PANI/GO/Azo composites were measured in the frequency range $5 \times 10^{-2} \sim 10^{5} \mathrm{~Hz}$. As shown in Figure 9, the semicircle is related to the impedance of the electrochemical reaction, while the oblique line in the low-frequency region is related to the solid phase diffusion of the ions in the electrode [45]. The charge transfer resistance (Rct) which occurs at the electrode/electrolyte interfaces during the electrochemical reaction and the Rct values of PANI/GO and PANI/GO/Azo were $1.312 \Omega$ and $1.407 \Omega$, respectively. This result indicates that the PANI/GO/Azo composite has higher charge transfer efficiency. Also, the slope of the impedance curve of PANI/ $\mathrm{GO} / \mathrm{Azo}$ is higher than the PANI/GO composite, which indicates that the PANI/GO/Azo composite has high ionic conductivity.

The Nyquist plots of the PANI/GO/Azo composite upon UV irradiation for different times are displayed in Figure 11(b). As irradiation time increasing, the Rct values of PANI/GO/Azo are all around $1.3 \Omega$, and the slope of the impedance curve of PANI/GO/Azo reduces gradually in the low-frequency region. This phenomenon shows that transPANI/GO/Azo has higher ionic conductivity than the cis-

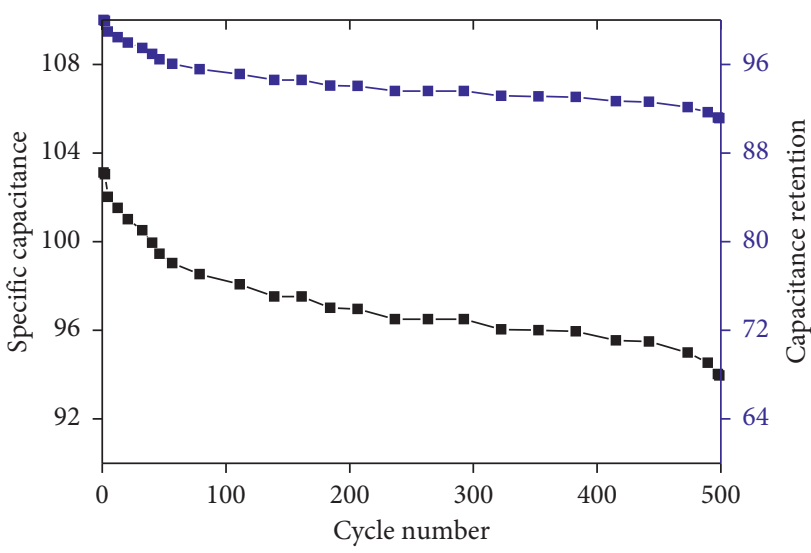

FIGURE 10: The capacitance and the capacitance retention in the relation of the cycling number for the PANI/GO/Azo composite (no UV irradiation) electrode.

PANI/GO/Azo composite. As Figure 11(b) shows, without UV irradiation, the PANI/GO/Azo composite has the highest slope of the Nyquist plots in the low-frequency region, which indicated it has the best capacitance. Meanwhile, the result was corroborated by the charge-discharge curves of the samples obtained at a current density of $1 \mathrm{Ag}^{-1}$. All the results showed that the capacitance performance of the PANI/GO/Azo composite could be adjusted by UV irradiation.

\section{Conclusions}

In summary, a new photosensitive hybrid material-PANI/ GO/Azo composite-was prepared, and its electrochemical performance was investigated. It was found that the electrochemical properties of the PANI/GO/Azo composite (without UV irradiation) were superior to the PANI/GO composite, such as significantly higher capacitance, charge, and discharge time prolonging, and the material impedance changes. Moreover, the PANI/GO/Azo composite has excellent photosensitive electrochemical properties, and its 


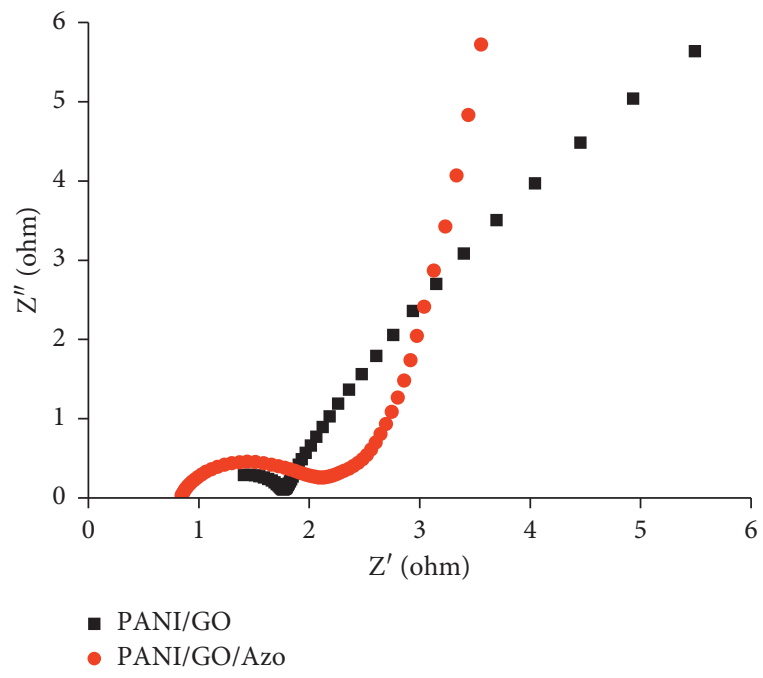

(a)

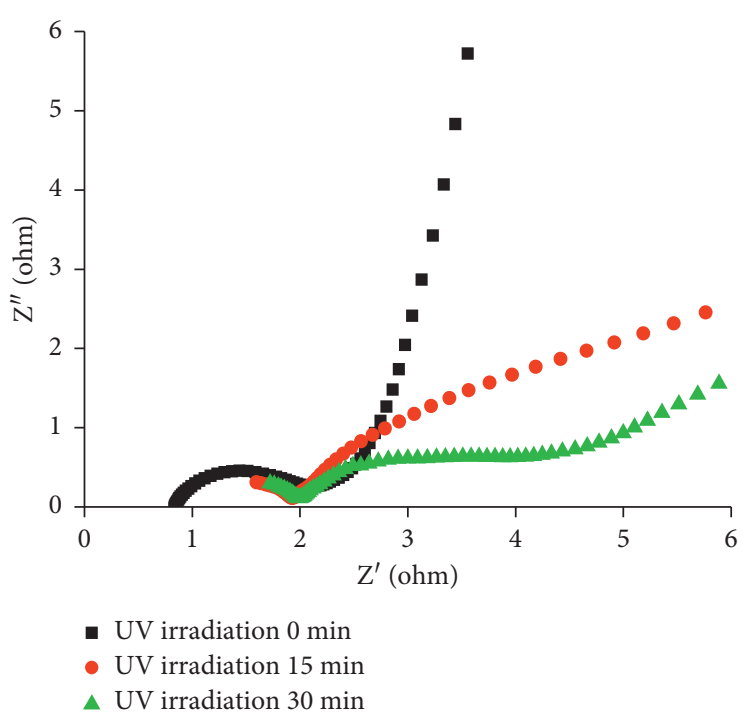

(b)

FIgure 11: The Nyquist plots of (a) PANI/GO and PANI/GO/Azo composites; (b) PANI/GO/Azo composites at different UV irradiation times.

electrochemical performance can be significantly adjusted by UV irradiation. Therefore, the PANI/GO/Azo composite has a great potential to be used as an adjustable electrode material for practical application in supercapacitors.

\section{Data Availability}

The data used to support the findings of this study are available from the corresponding author upon request.

\section{Conflicts of Interest}

The authors declare no conflicts of interest.

\section{Acknowledgments}

This work was supported by the National Natural Science Foundation of China (Grant no. 11872001) and the Excellent Youth Foundation of Anhui Scientific Committee (Grant no. 1808085 J30).

\section{References}

[1] M. Manoj, K. M. Anilkumar, B. Jinisha, and S. Jayalekshmi, "Polyaniline-graphene oxide based ordered nanocomposite electrodes for high-performance supercapacitor applications," Journal of Materials Science: Materials in Electronics, vol. 28, no. 19, pp. 14323-14330, 2017.

[2] A. G. Tabrizi, N. Arsalani, H. Namazi, and I. Ahadzadeh, "Vanadium oxide assisted synthesis of polyaniline nanoarrays on graphene oxide sheets and its application in supercapacitors," Journal of Electroanalytical Chemistry, vol. 798, pp. 34-41, 2017.

[3] L. Tang, Z. Yang, F. Duan, and M. Chen, "Hierarchical architecture of ultrashort carbon nanotubes/polyaniline nanocables coated on graphene sheets for advanced supercapacitors," Journal of Materials Science: Materials in Electronics, vol. 28, no. 21, pp. 15804-15818, 2017.
[4] T.-W. Chang, L.-Y. Lin, P.-W. Peng, Y. X. Zhang, and Y.-Y. Huang, "Enhanced electrocapacitive performance for the supercapacitor with tube-like polyaniline and graphene oxide composites," Electrochimica Acta, vol. 259, pp. 348-354, 2018.

[5] A. G. Tabrizi, N. Arsalani, A. Mohammadi, L. S. Ghadimi, I. Ahadzadeh, and H. Namazi, "A new route for the synthesis of polyaniline nanoarrays on graphene oxide for high-performance supercapacitors," Electrochimica Acta, vol. 265, pp. 379-390, 2018.

[6] W. Wang, J. Yan, J. Liu et al., "Self-healing polyaniline-graphene oxides based electrodes with enhanced cycling stability," Electrochimica Acta, vol. 282, pp. 835-844, 2018.

[7] N. R. Dywili, A. Ntziouni, C. Ikpo et al., "Graphene oxide decorated nanometal-poly (anilino-dodecylbenzene sulfonic acid) for application in high performance supercapacitors," Micromachines, vol. 10, no. 2, pp. 1-17, 2019.

[8] Q. Jiang, Y. Shang, Y. Sun et al., "Flexible and multi-form solid-state supercapacitors based on polyaniline/graphene oxide/CNT composite films and fibers," Diamond and Related Materials, vol. 92, pp. 198-207, 2019.

[9] T. Yu, P. Zhu, Y. Xiong et al., "Synthesis of microspherical polyaniline/graphene composites and their application in supercapacitors," Electrochimica Acta, vol. 222, pp. 12-19, 2016.

[10] M. O. Ansari, R. Kumar, S. A. Ansari et al., "Anion selective pTSA doped polyaniline@graphene oxide-multiwalled carbon nanotube composite for $\mathrm{Cr}(\mathrm{VI})$ and Congo red adsorption," Journal of Colloid and Interface Science, vol. 496, pp. 407-415, 2017.

[11] H. Xu, J. Tang, Y. Chen, J. Liu, J. Pu, and Q. Li, “ $\mathrm{Zn}^{2+}$-Doped polyaniline/graphene oxide as electrode material for electrochemical supercapacitors," Journal of Electronic Materials, vol. 46, no. 10, pp. 6150-6157, 2017.

[12] R. Kötz and M. Carlen, "Principles and applications of electrochemical capacitors," Electrochimica Acta, vol. 45, no. 15-16, pp. 2483-2498, 2000.

[13] W.-C. Chen and T.-C. Wen, "Electrochemical and capacitive properties of polyaniline-implanted porous carbon electrode for supercapacitors," Journal of Power Sources, vol. 117, no. 12, pp. 273-282, 2003. 
[14] D. Huang, Z. Yang, X. Li et al., "Three-dimensional conductive networks based on stacked SiO2@graphene frameworks for enhanced gas sensing," Nanoscale, vol. 9, no. 1, pp. 109-118, 2017.

[15] N. Hu, L. Zhang, C. Yang et al., "Three-dimensional skeleton networks of graphene wrapped polyaniline nanofibers: an excellent structure for high-performance flexible solid-state supercapacitors," Scientific Reports, vol. 6, no. 1, p. 19777, 2016.

[16] Y. Ma, D. Zhao, Y. Chen et al., "A novel core-shell polyaniline/ graphene oxide/copper nanocomposite for high performance and low-cost supercapacitors," Chemical Papers, vol. 73, no. 1 , pp. 119-129, 2019.

[17] B. Vedhanarayanan, T.-H. Huang, and T.-W. Lin, "Fabrication of 3D hierarchically structured carbon electrode for supercapacitors by carbonization of polyaniline/carbon nanotube/graphene composites," Inorganica Chimica Acta, vol. 489, pp. 217-223, 2019.

[18] Z. Qiang, J. Chen, F. Luo et al., "Vertically oriented polyaniline-graphene nanocomposite based on functionalized graphene for supercapacitor electrode," Applied Polymer Science, vol. 134, no. 19, 2017.

[19] J. Li, H. Xie, and Y. Li, "Electrochemical performance of graphene oxide/polyaniline composite for supercapacitor electrode," Journal of Nanoscience and Nanotechnology, vol. 15, no. 4, pp. 3280-3283, 2015.

[20] K. Chen, L. Chen, Y. Chen, H. Bai, and L. Li, "Three-dimensional porous graphene-based composite materials: electrochemical synthesis and application," Journal of Materials Chemistry, vol. 22, no. 39, pp. 20968-20976, 2012.

[21] L. Zhe-Fei, Z. Hangyu, L. Qi et al., "Fabrication of highsurface-area graphene/polyaniline nanocomposites and their application in supercapacitors," ACS Applied Materials \& Interfaces, vol. 5, no. 7, pp. 2685-2691, 2013.

[22] L. Jing, M. Qiang, H. Gu et al., "Three-dimensional graphenepolyaniline hybrid hollow spheres by layer-by-layer assembly for application in supercapacitor," Electrochimica Acta, vol. 173, pp. 184-192, 2015.

[23] J. J. Wie, S. Chatterjee, D. H. Wang, L.-S. Tan, M. Ravi Shankar, and T. J. White, "Azobenzene-functionalized polyimides as wireless actuators," Polymer, vol. 55, no. 23, pp. 5915-5923, 2014.

[24] X. Zhang, Y. Feng, D. Huang, Y. Li, and W. Feng, "Investigation of optical modulated conductance effects based on a graphene oxide-azobenzene hybrid," Carbon, vol. 48, no. 11, pp. 3236-3241, 2010.

[25] X. Zhang, Y. Feng, P. Lv, Y. Shen, and W. Feng, "Enhanced reversible photoswitching of azobenzene-functionalized graphene oxide hybrids," Langmuir, vol. 26, no. 23, pp. 18508-18511, 2010.

[26] S. Chen, L. Bao, E. Ou, C. Peng, W. Wang, and W. Xu, “A cationic azobenzene-surfactant-modified graphene hybrid: unique photoresponse and electrochemical behavior," Nanoscale, vol. 7, no. 46, pp. 19673-19686, 2015.

[27] D. Hu, J. Lin, S. Jin et al., "Synthesis, structure and optical data storage properties of silver nanoparticles modified with azobenzene thiols," Materials Chemistry and Physics, vol. 170, pp. 108-112, 2016.

[28] H. Guo, J. Yang, J. Zhou, L. Zeng, L. Zhao, and B. Xu, "Photoresponsive self-assembly of a $\beta$-cyclodextrin derivative with an azobenzene terminal group in water," Dyes and Pigments, vol. 149, pp. 626-632, 2018.

[29] A. A. Beharry and G. A. Woolley, "Azobenzene photoswitches for biomolecules," Chemical Society Reviews, vol. 40, no. 8, p. $4422,2011$.
[30] H. M. D. Bandara and S. C. Burdette, "Photoisomerization in different classes of azobenzene," Chemical Society Reviews, vol. 41, no. 5, pp. 1809-1825, 2012.

[31] H. Zhou, C. Xue, P. Weis et al., "Photoswitching of glass transition temperatures of azobenzene-containing polymers induces reversible solid-to-liquid transitions," Nature Chemistry, vol. 9, no. 2, pp. 145-151, 2016.

[32] K. G. Yager and C. J. Barrett, "Novel photo-switching using azobenzene functional materials," Journal of Photochemistry and Photobiology A: Chemistry, vol. 182, no. 3, pp. 250-261, 2006.

[33] S. Hagen, F. Leyssner, D. Nandi, M. Wolf, and P. Tegeder, "Reversible switching of tetra-tert-butyl-azobenzene on a $\mathrm{Au}(111)$ surface induced by light and thermal activation," Chemical Physics Letters, vol. 444, no. 1-3, pp. 85-90, 2007.

[34] S. Kurihara, A. Sakamoto, D. Yoneyama et al., "Photochemical switching behavior of liquid crystalline polymer networks containing azobenzene molecules," Macromolecules, vol. 31, no. 14, pp. 6493-6498, 1999.

[35] A. S. Kumar, T. Ye, T. Takami et al., "Reversible photoswitching of single azobenzene molecules in controlled nanoscale environments," Nano Letters, vol. 8, no. 6, pp. 1644-1648, 2008.

[36] J. Wang, Y. Shi, K. Yang, J. Wei, and J. Guo, "Stabilization and optical switching of liquid crystal blue phase doped with azobenzene-based bent-shaped hydrogen-bonded assemblies," RSC Advances, vol. 5, no. 82, pp. 67357-67364, 2015.

[37] S. Chen, C. Wang, Y. Yin, and K. Chen, "Synthesis of photoresponsive azobenzene molecules with different hydrophobic chain length for controlling foam stability," RSC Advances, vol. 6, no. 65, pp. 60138-60144, 2016.

[38] C. Y. Lin, C. S. Chang, J. H. Lin et al., "Optical controlled graphene-based nonvolatile ternary-logic transistor with azobenzene copolymer," Applied Physics Letters, vol. 102, no. 1, p. 487, 2013.

[39] C. Shu, W. Wang, and W. Xu, "A photoresponsive azobenzene-graphene-gold nanocomposite using cationic azobenzene-surfactant as stabilizers and its electrochemical behavior," Materials Letters, vol. 180, pp. 196-199, 2016.

[40] D. R. Kizhisseri, G. Venugopal, C. L. Lekshmi, K. Joseph, and S. Mahesh, "Photoresponse modulation of reduced graphene oxide by surface modification with cardanol derived azobenzene," New Journal of Chemistry, vol. 42, no. 22, pp. 18182-18188, 2018.

[41] P. Weis, D. Wang, and S. J. M. Wu, "Visible-light-responsive azopolymers with inhibited $\pi-\pi$ stacking enable fully reversible photopatterning," Macromolecules, vol. 49, no. 17, pp. 6368-6373, 2016.

[42] L. Haiying and L. Zhongfan, "A convenient synthesis of novel mercapto-ended azobenzene derivatives," Synthetic Communications, vol. 28, no. 20, pp. 3779-3785, 1998.

[43] Y. Li and Y. Zheng, "Preparation and electrochemical properties of polyaniline/reduced graphene oxide composites," Journal of Applied Polymer Science, vol. 135, no. 16, p. $46103,2018$.

[44] A. Eftekhari and P. Jafarkhani, "Curly graphene with specious interlayers displaying superior capacity for hydrogen storage," The Journal of Physical Chemistry C, vol. 117, no. 48, pp. 25845-25851, 2013.

[45] J. An, J. Liu, Y. Zhou et al., "Polyaniline-grafted graphene hybrid with amide groups and its use in supercapacitors," The Journal of Physical Chemistry C, vol. 116, no. 37, pp. 1969919708, 2012. 